\title{
Isolation and Structural elucidation of novel bioactive molecule- Coumarin from traditionally used Medicinal Plant- Ceropegia juncea (Roxb.)
}

\author{
Sudha Karayil, Subhash Chandran K.P, Sudeesh P.S. \&Veraiah.K \\ Department of Biotechnology\& Department of Chemistry \\ Acharya Nagarjuna University Guntur, AP,India
}

\begin{abstract}
Coumarins have attracted the attention in recent years because of their diverse pharmacological properties and these diverse properties are mainly due to their structural variability and types of substitutions in their basic structure. Ceropegia juncea (Roxb.) is the plant, which belongs to the Asclepiadoideae, a subfamily belongs to the family Apocyanaceae was selected and investigated for its phytocompounds especially coumarin compounds. There was limited work on biologically active molecule, coumarin in the selected medicinal plant Ceropegia juncea (Roxb.). A novel coumarin, (E)-5-(4-methyl-2-oxo-2H-chromen-8-yl)-2-(4-methylbenzyl)-3oxopent-4-enenitrile was isolated and the structure of the compound is studied by spectral data. Biological functions of coumarins depend on their functional groups attached. 7-hydroxy and methyl coumarins exhibit dynamic therapeutical properties. In the present study, methyl coumarin is obtained from the plant extract. Further pharmacological activities have to be studied for this novel compound for future drug lead.
\end{abstract}

Key words: Coumarin, Ceropegia juncea, TLC, HPLC, Asclepiadoideae.

\section{Introduction:}

Natural products had been indispensably used by many cultures and traditions from thousands of years ${ }^{1}$.Traditional knowledge systems have given clues to the discovery of valuable drugs ${ }^{2}$. Use of indigenous drugs from plant origin forms a major part of complementary traditional medicine. In recent times, focus on plant research was drawn all over the world and a large body of evidence has collected to show immense potential of medicinal plants used in various traditional systems.

Several of Scientific investigations have proved and high-lighted the importance and contributions of many plant families. Among different families studied, Asclepidaceae, now called Asclepiadoideae a subfamily belongs to the family Apocyanaceae having different species of plants with wide therapeutical properties is selected for study. Asclepiadoideae is the largest cosmopolitan family having genera of 177 and nearly 3000 species $^{3-4}$ and are well known for their ethnobotanical and ethnomedicinal importance.

Ceropegia juncea (Roxb.) is the one in this family having wide medicinal properties and is being used in different traditional medical systems by tribal people for curing different ailments. Ceropegia juncea (Roxb.) was also claimed as one of the Soma plant ${ }^{5}$. Based on the importance of the ethnomedicinal and therapeutical properties through ancient and ethno medicinal literature, the plant Ceropegia juncea (Roxb.) was selected and investigated for its phytocompounds especially coumarin compounds. There was limited work on phytochemicals in the selected medicinal plant Ceropegia juncea (Roxb.). There were no reports on coumarin based compounds in Ceropegia juncea (Roxb.).

Coumarins are a group of plant-derived polyphenolic compounds. They belong to the benzopyrone family and possess a wide range of pharmaceutical applications including anti inflammatory, anti proliferative, anti coagulant, anti carcinogenic, cytoprotective, hepatoprotective and modulatory functions etc, which may be translated into therapeutic potential for multiple diseases ${ }^{6}$. Several natural and synthetic coumarins and derivatives, such as coumarin glycosides, possess potent biological activities. Coumarin, a 1, 2- benzopyrone is the simplest compound of a large class of naturally occurring phenolic substances made of fused benzene and a pyrone ring ${ }^{7}$. They have recently drawn much attention due to their broad pharmacological activities. The recognition of important structural features within coumarin family was crucial for the design and development of new analogues with much improved activity.

The Coumarins have long been recognized to possess anti-inflammatory, antioxidant, antiallergic, hepatoprotective, antithrombotic, antiviral, and anticarcinogenic, analgesic activities ${ }^{8-9}$. Coumarins have attracted the attention in recent years because of their diverse pharmacological properties ${ }^{10}$ and these diverse properties are mainly due to their structural variability and types of substitutions in their basic structure ${ }^{11}$. Other than the medicinal properties, Coumarin has a history of importance in perfumery. It is the first synthetic substance to be used in a fragrance - Fougère Royale (Houbigant) and found in essential oils and is used in food industries for $\operatorname{aroma}^{12}$. 
Some naturally occurring Coumarin derivatives include umbelliferone (7-hydroxycoumarin), aesculetin (6,7-dihydroxycoumarin), herniarin (7-methoxycoumarin), psoralen and imperatorin.4-Phenylcoumarin was the backbone of the neoflavones ${ }^{13-14}$. Coumarins have been identified from a number of natural sources, especially green plants. Coumarins are found free or as heterosides in many dicotyledonous families, including the Asteraceae, Fabiaceae, Rosaceae, Rubiaceae and Rutaceae ${ }^{15-16}$.

\section{Materials \& Methods:}

In the present study, the plant Ceropegia juncea(Roxb.) is selected for the isolation of biologically active phytocompound. The whole plant is taken (without flowers) for extraction, done by TLC and the phytoconstituents (mixture of compounds) found in the plant sample is separated by Column chromatography.

\section{Extraction of Coumarin:}

Coumarin was extracted from the plant crude. Plant material was air dried, powdered and extracted with $80 \%$ ethanol at 650C for 5 hours in a water bath shaker. The supernatant was centrifuged twice for 10 minutes at $10000 \mathrm{rpm}$ and filtered through $0.22 \mu$ filter and the filtrate is evaporated by rotary evaporator at $40 \mathrm{c}$ under reduced pressure.** The clear supernatant was taken for Thin Liquid Chromatography analysis.

\section{Thin layer chromatographic (TLC)}

Detection of Coumarin:

For rapid screening of coumarin in the crude extracts, TLC was performed by adopting the standard method. Crude extracts of the samples were run along with the standard coumarin in silica gel 60 F254 plates. Crude was extracted into following solvent in the ratio of Ethyl acetate: Pet Ether: Methanol: water 5:5:7:3. Pale yellow color crude was obtained. It was dried at room temperature. The run time was 1.6 hours. The crude extracts whose Rf values and UV light florescence matched with standard coumarin were further taken for quantification by HPLC.

\section{Estimation of Total Coumarin Content by HPLC: Sample preparation:}

10 grams of sample was extracted in methanol by Soxhlet extraction continuously up to 48 hours. 100 $\mathrm{mg}$ of crude was taken in to mobile phase and placed in $100 \mathrm{ml}$ mobile phase. Sample injected and amount of total Coumarin from calibration curve was plotted.

Structure Analysis: Structure analyses of compounds were done by using the spectral data.

\section{HPLC Quantification of Coumarin:}

Coumarin was analyzed by Reverse phase HPLC on C-18 column $(250 \times 4.6 \mathrm{~mm} 5 \mu)$. The HPLC was flushed with $100 \%$ methanol and changed to the required mobile phase. Benzopyrone (Sigma, 99\% HPLC purified) standard sample was procured from Sigma Chemicals. The run time of coumarin was 24 minutes. For every five runs, the HPLC was re-standardized using the coumarin standard. The HPLC parameters were set following with standard methods with appropriate modifications

Mobile phase condition for HPLC: methanol-acetonitrile-phosphate buffer (PH 4.8; 1mM) (22.5:15:62.5, $\mathrm{v} / \mathrm{v} / \mathrm{v})$.

Stationary phase: RP-C18 $(250 \times 4.6 \mathrm{~mm} / 5 \mu)$

Flow rate: $0.5 \mathrm{ml}$ per minute

Detection range: $274 \mathrm{~nm}-365 \mathrm{~nm}$

\section{Preparation of calibration curve:}

Benzopyrone was taken as standard compound for coumarin estimation in given sample.1000 ppm standard solution was prepared with Benzopyrone. From stock solution 5,10,20,40,80,160,320,640 ppm solution were prepared and injected in to HPLC for calibration curve. Calibration curve was constructed by plotting the amount of coumarin injected $(\mu \mathrm{g})$ of the reference substance versus the peak area in the chromatogram. Coumarin levels were quantified in the plant samples by using the regression of peak areas against the standard coumarin and expressed as micrograms per gram dry weight.

\section{Spectral Studies:}

$10 \mathrm{ppm}$ concentrated sample solution are prepared in Methanol as a solvent. The structures of the compounds were established by spectral data and by comparison with the literature data. The compounds were 
Isolation and Structural elucidation of novel bioactive molecule- Coumarin from traditionally...

isolated by chromatographic techniques. The structural elucidation of these compounds was based on spectroscopic data, especially IR, 1H- NMR and 13C- NMR, Mass, 2D COSY, HMBC, HSQC.

\section{Structure of Coumarin:}

\section{Results:}

The obtained structure of coumarin from Ceropegia juncea (Roxb.):

(E)-5-(4-methyl-2-oxo-2H-chromen-8-yl)-2-(4-methylbenzyl)-3-oxopent-4-enenitrile

The nomenclature used for chemical compound is in ccordance with the nomenclature rules formulated by IUPAC.

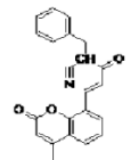

Ionization Mass spectrometry indicated the molecular ion peak $\mathrm{m} / \mathrm{z}=360$. High Resolution Chemical Ionization Mass spectrometry (HRCIMS) and High Resolution Electrom Impact Mass spectrometry ( HREIMS) supported the molecular formula $\mathrm{C}_{21} \mathrm{H}_{15} \mathrm{~N} \mathrm{O}_{3}$ for the Coumarin. The molecular ion fragment was observed at $\mathrm{m} / \mathrm{z}$ 343. The major fragment ions at $\mathrm{m} / \mathrm{z} 334,292,262,219,177,129$ and 100. Mass spectra support the molecular formula. The number of protons from integration in the $\mathrm{H}^{1} \mathrm{NMR}$ spectrum and carbons from $\mathrm{C}^{13}$ NMR matched with number of protons and carbons presented in the molecular formula. The structure of Coumarin was established by the analysis of $\mathrm{H}^{1} \mathrm{NMR}, \mathrm{C}^{13} \mathrm{NMR}$, gCOSY, g HMBC, gHSQC, IR and 2D.

\section{IR Analysis:}

The Wave number indicates $948 \mathrm{C}-\mathrm{N}$ bonds, 1062 represent C-O, 1199 indicates $\mathrm{C}-\mathrm{C}$ bonds between $\mathrm{C}_{13}, \mathrm{C}_{22}$, and $\mathrm{C}_{14}$ in IR spectra. 2924 indicates $\mathrm{C}-\mathrm{H}$ bond and 2969 indicates $\mathrm{C}-\mathrm{H}$ bond, 3052 indicates $\mathrm{N}-\mathrm{H}$, 3478- indicates $\mathrm{O} \mathrm{H}$, and 2902 represents $\mathrm{C}=\mathrm{C}, 1625$ indicates $\mathrm{C}=\mathrm{O}$.

\section{$C^{13}$ NMR Analysis:}

The chemical shift value $\delta 160,159,158$ indicates the $\mathrm{C} 1, \mathrm{C} 5, \mathrm{C} 12, \delta 110-119$ indicates C4-C9; Chemical shift of 142,145 , and 147 showed C14-C19 carbons. $\delta$ value 436 indicates C10 and C11 carbons. CNMR values indicating the position of Carbon22.

\section{$H^{1}$ NMR Analysis:}

The chemical shift $\delta$ value $6.8,6.7$ indicates the presence of $\mathrm{H}$ on $\mathrm{C} 4, \mathrm{C} 5, \mathrm{C} 6, \mathrm{C} 7, \mathrm{C} 8, \mathrm{C} 9$ and $\mathrm{C} 14$ C19. $\delta$ value of 3.2 indicates the presence of $\mathrm{H}$ on $\mathrm{C} 10, \mathrm{C} 11 . \delta$ of 8.3 showed the protons on $\mathrm{C} 21 ., 8.3$ indicate the hydrogen on $\mathrm{C} 22$.

\section{Total amount of coumarin by HPLC:}

Sample peak area $=18548.2$

Amount of Coumarin is $18548.2=652.6 \mathrm{x}+11837.8=10.258 \mu \mathrm{g} / \mathrm{ml}$, appeared as pale $\quad$ yellow coloured crystals

\section{Discussion:}

All the spectral studies supported the structures of coumarin. There were no earlier reports on the presence of coumarins in Ceropegia juncea(Roxb.) There are several reports on coumarins in different medicinal plants of different families. The compounds include 7-hydroxy coumarins and 4-methyl coumarins with cyano, keto, benzene, methyl functional groups. Coumarin derivatives possess wide pharmaceutical and therapeutical properties mainly based on their substitution of functional groups. Recent studies proved that 7-hydroxy and methyl coumarins possess potent anticancer activities.

There are very few reports regarding the presence of coumarin compounds in the families, Asclepidaceae and Apocynaceae. The literature survey revealed that the coumarins were isolated from the plant 
Isolation and Structural elucidation of novel bioactive molecule- Coumarin from traditionally...

Secamone afzelii (Asclepidaceae) ${ }^{17}$. Johns et al. ${ }^{18}$ reported the high yield of unsubstituted coumarins in leaves and barks of Lepiniopsis ternatensis Val.( Apocynaceae).

Coumarin is a compound found in nature at high levels in some plants, essential oils, particularly cinnamon bark oil (7000 ppm), cinnamon leaf oil (40 $600 \mathrm{ppm}(\mathrm{mg} / \mathrm{kg}))$ and cassia leaf oil (17 000-87 300 $\mathrm{ppm}$ ). It is also found in Mexican vanilla extracts ${ }^{19-20}$. It was subsequently identified in a large number of plants belonging to many different families. Broad spectrums of coumarin derivatives (present both in the Free State and as glucosides) have also been found in many plants. To date about 1300 coumarin compounds have been identified, principally as secondary metabolites in green plants ${ }^{6}$. The hydroxyl coumarins are typical phenolic compounds and therefore act as potent metal chelators and free radical scavengers ${ }^{10}$. The pharmacological, biochemical properties and therapeutic applications of coumarins depend upon their pattern of substitution ${ }^{10-11}$. Several beneficial pharmacological effects are implicated to have 4-Methyl coumarins ${ }^{21-22}$. Similar reports were many in different medicinal plants and different coumarin structures. From the present results, the obtained novel 4-methyl coumarin compound from Ceropegia juncea (Roxb.) is to be analysed for biological and pharmacological activities and it could be source for potential drug candidates for future use. New chemical substances found in plants will continue to serve as models for novel synthetic and will prove to be increasingly important in the future. The pharmacological activities has to be investigated for this novel compound.

\section{Reference:}

[1]. G.Samuelsson. "DrugsofNaturalOrigin”.ATextbookofPharmacognosy,5thSwedishPharmaceuticalPress.Stockholm,2004

[2]. E.T.Buenz,D.J.Schnepple,B.A.Bauer,P.L.Elkin,J.M.Riddle,T.J.Motely."Techniques:BioprospectinghistoricalHerbaltextsbyhuntingf ornewleadsinOldtomes".TrendsTrends Pharmacol Sci,25(9):2004,494-8.

[3]. Meve.Speciesnumbersandprogressinasclepiadtaxonomy.Kewbulletin.57:2002b,459-464.

[4]. B.A.Jadeja,D.C.Bhatt,N.K."EthnobotanicalsignificanceofAsclepiadaceaeinBardahillsof Gujarat, India".OdedraPlantArchives.4:2004,459-461.

[5]. AlamMuzaffer,K.Sathavasan,S.AliUsman,V.N.K.Ramadas.Chelladurai.AnalyticalValuesofSarcostemmaacidumandCeropegiajunce atheSomaPlantsinAyurveda".BMEBR3:1982,238-243.

[6]. J.Hoult,M.Payd.Pharmacologicalandbiochemicalactionsofsimplecoumarins: naturalproductswiththerapeuticpotentialGen.Pharmacol,27,1996,713-722

[7]. G.J.Keating,R.O'Kennedy,."InThechemistryandoccurrenceofcoumarins”O'Kennedy,R.;ThornesR.D.Eds.;JohnWiley\&Sons.WestSu ssex,England,1997,pp.23-64. 8.T.O.Soine,Naturallyoccuringcoumarinsandrelatedphysiologicalactivities.J.Pharm.Sci.53:1964, 231264.

[8]. R.O'Kennedy,andR.D.Thornes.Coumarins-Biology,ApplicationsandModeofAction,2003, JohnWiley\&SonsLtd.,Chichester

[9]. Irenakostova.):"SyntheticandNaturalcoumarinsascytotoxicagents".Curr.Med.Chem.5:2005,29-

[10]. M.PatelRajesh,PatelNatwar.):"Invitroantioxidantactivityofcoumarin compoundsby DPPH, SuperoxideandNitricoxidefreeradicalscavengingmethods".JournalofAdvancedPharmacyEducation\&Research,1:2011,52-68.

[11]. U.Matern,P.Luer,D.Kreusch,Biosynthesisofcoumarins.Comprehensive NaturalProductsChemistry.1: 1999,623-637.

[12]. F.Borges,F.Roleira,N.Milhazes,L.Santana,E.Uriarte.Curr.Med.Chem.12:2005,887.

[13]. I.Weinmann.Historyofthedevelopmentandapplicationsofcoumarinandcoumarin-relatedcompounds. 1997,Pp.1-

[14]. 22.In:O’Kennedy\&Thornes.

[15]. U.Matern,P.Luer,D.Kreusch.Biosynthesisofcoumarins.1999,Pp.623- 637.In: Barton, D.,Nakanishi,K.,MethCohn,O.andSankawa,U.(eds.):Elsevier ScienceLtd.,Oxford,UK.

[16]. ZabriHerve,KabranGuyRoger,KodjoCharles.): "Purificationcolumnonsilicaandchemicalcharacterizationofacoumarinisolatedfrommet hanolexcerptofplantSecamoneafzelia(Asclepiedaceae) fromAbidjan-Ivorycoast”.Trabi Fezan. Journal of

[17]. Animal\&PlantSciences,2:2009,182-185.

[18]. S.R.Johns,A.Lamberton,J.R.Price,A.A.Sioumis. “IdentificationofCoumarinsisolatedfrom LepiniopsisTernatensis(Apocyanaceae),Pterocaulonsphacelatum(Compositae)andMelicopemelanophloia(Rutaceae)".Aust.J.Chem.2 $1: 1968,3079-80$

[19]. G.Sullivan.ExaminationofMexicanvanillaextractsforcoumarinadulteration.III.Quantitativedeterminationofcoumarin.Vet.hum.Toxico 1.23:1981,249-251.

[20]. R.J.Marles,C.M.Compadre,N.R.Farnsworth.Coumarininvanillaextracts:itsdetectionandsignificance. Econ.Botany,41:1987,41-47

[21]. S.Takeda,M.J.Aburada.Pharmacobiodyn.4:1981,724

[22]. A.A.Deana,G.E.Stokker,E.M.Schultz,R.L.Smith,E.J.Cragoe,H.F.Russo,L.S.Watson,J.Med.Chem., 26:1983580.

HPLC Report

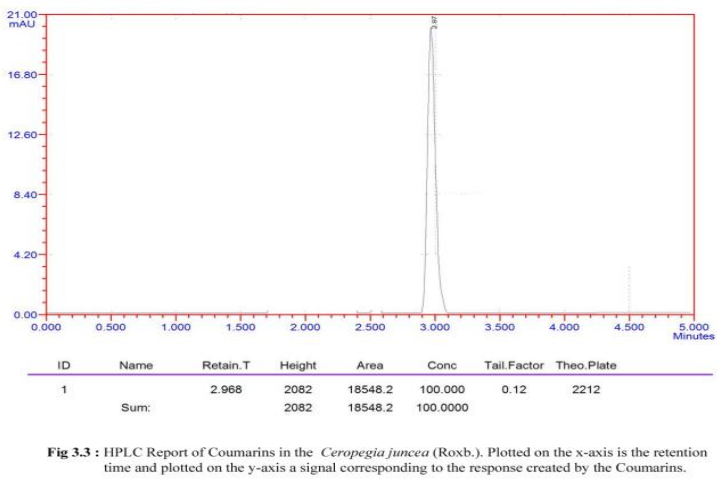

